\title{
Revealing the Native Molecular Architecture of the Nuclear Periphery using Cryo- Focused-Ion-Beam Milling, Light Microscopy and Electron Tomography
}

\author{
Elizabeth Villa $^{1}$, Reika Watanabe ${ }^{1}$, Robert Buschauer ${ }^{1,2}$, Kanika Khanna ${ }^{1}$, Vinson Lam ${ }^{1}$, Jitin Singla ${ }^{3}$, \\ Frank Alber ${ }^{3}$ \\ 1. Division of Biological Sciences, University of California San Diego, La Jolla, USA. \\ 2. Gene Center, Ludwig Maximillian University, Munich, Germany. \\ 3. Department of Biological Sciences, University of Southern California, Los Angeles, USA.
}

Understanding the architecture of the nuclear periphery remains one of the biggest challenges in biology, with the nucleus presenting an impregnable frontier for structural studies. Molecular complexes and DNA that conform this region of the cell are deeply rooted in their environment, and in many cases, such as chromatin, any form of fixation, staining, and extraction is thought to alter their structure.. A variety of imaging techniques intend to reveal the architecture of the nuclear periphery, with a strong focus on chromatin. Structural biology provides deep insight into isolated complexes devoid of their natural context, with the latter often determining their conformation, composition, and function. Light microscopy has many advantages but relies on in vivo tagging but lacks detailed substructure information. Traditional electron microscopy allows detailed ultrastructural analyses but relies on fixation, embedding, and staining, often compromising structural integrity and hampering interpretation at the molecular level. Thus, a major challenge remains to combine structural analyses of chromatin with native preservation of its $3 \mathrm{D}$ folding and positioning within the nuclear landscape. Cryo-electron microscopy has undergone a resolution revolution due to technological advances. In its single-particle modality for structural biology, it has become a central technique capable of achieving atomic-resolution structures even flexible and transient complexes. In the modality of CET, structures can be visualized within 3-D images of cells. CET was limited by the size of the objects $(<500 \mathrm{~nm})$ that can be examined in situ with molecular resolution. Using cryo-FIB milling of cells, we have eliminated this limitation (Fig. 1)[1,2], We have demonstrated the power of cryo-FIB/CET by visualizing the nuclear periphery of HeLa cells and determining the structural dynamics of large and complex machines such as the nuclear pore complex [3] and revealing how large viruses affect the cellular machinery of bacteria during infection by creating a nucleus-like compartment that separates transcription and translation [4].

Using cryo-focused ion beam (cryo-FIB) milling and cryo-electron tomography (CET), we obtain highresolution 3-D images of intact (cryo-frozen) cells that have not been fixed, permeabilized, or stained. The resulting data reveal the local architecture of chromatin at the single nucleosome level, along with the structures of individual architectural complexes. To characterize these structures, we will combine CET with rigorous computational methodologies that can perform large-scale template-free discovery of structurally unknown complexes. Using cryo-FIB milling and CET to obtain 3-D molecular landscapes of the nuclear periphery of mammalian cells, we demonstrate that CET can be leveraged into the highest resolution method to (a) extract the local architecture of native chromatin at the molecular level in situ (i.e., single and stretches of nucleosomes) and (b) we will show the potential to determine the in situ structures, abundance and nuclear locations of molecular complexes that organize the nuclear periphery irrespectively of whether their structure is known a priori. To identify instances of the macromolecular complexes of interest need to be identified within tomograms and averaged to increase resolution. Structures of most complexes in the nuclear periphery are unknown and therefore intractable by 
traditional template matching approaches. We begin by identifying regions of interest using correlative light and electron cryo-microscopy, and image these regions in high resolution using CET. To detect these structures in tomograms, we apply computational methods for large-scale template-free pattern mining, which determine and average frequently occurring complexes without relying on known structures.

\section{References:}

[1] E Villa, M Schaffer M, JM Plitzko JM, W Baumeister, Curr Opin Struct Biol (2013). P.771-7.

[2] RF Wagner, R Schampers, R Watanabe, H Persoon, et al. Nat. Protoc. (2017).Under revision.

[3] J Mahamid, S Pfeffer, M Schaffer, E Villa, R Danev, et al. Science (2016) p. 969-72.

[4] V Chaikeeratisak, K Nguyen, K Khanna, AF Brilot et al. Science (2017) p. 194-7.

[5] M Xu, IE Tocheva, Y Chang, JG Jensen, F Alber. arXiv:1512.09347 [q-bio.QM] (2016).

[6] The authors acknowledge funding from a NIH Director's New Innovator Award (to E.V.)
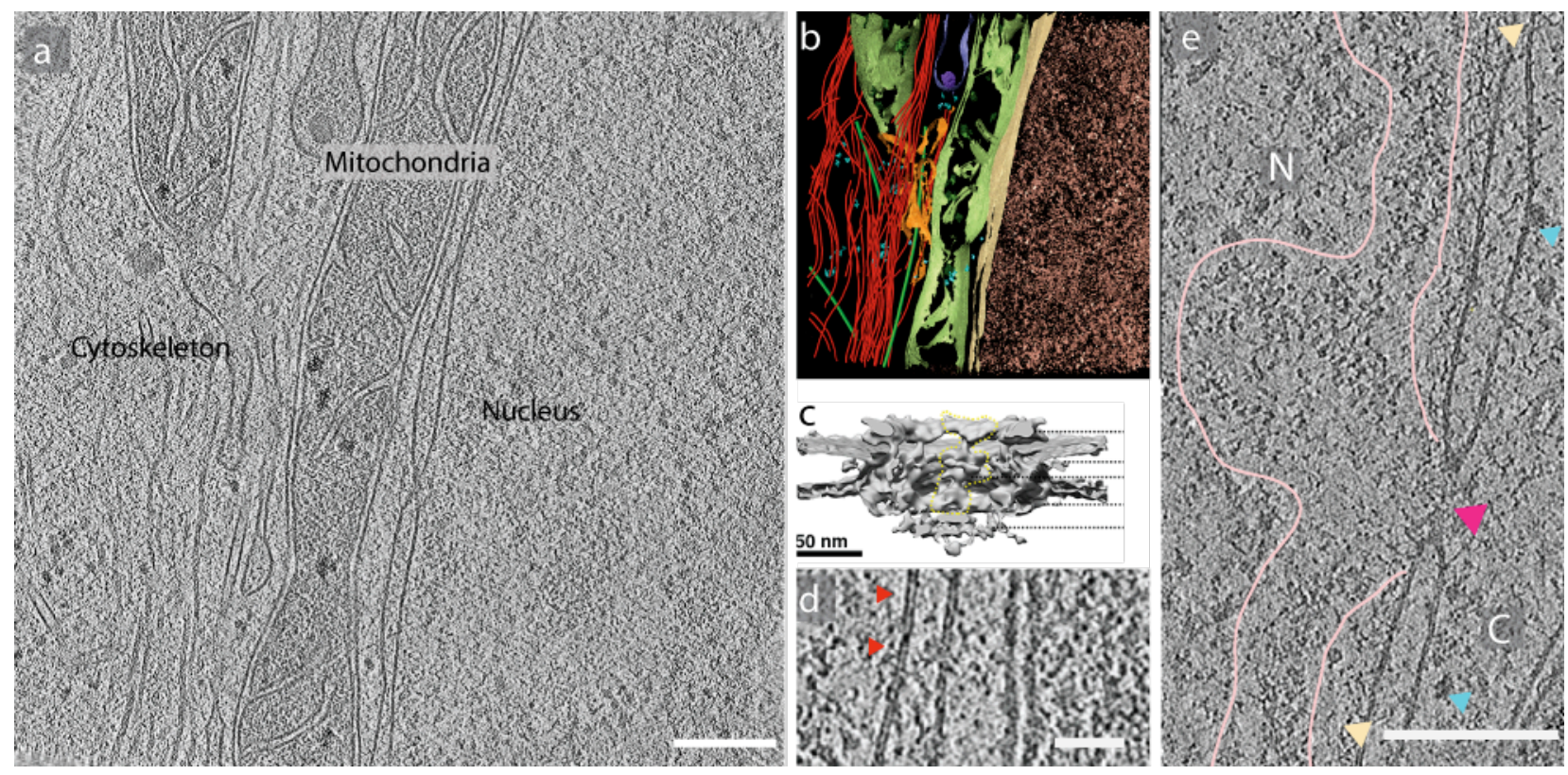

Figure 1. The power of cryo-FIB milling and CET to reveal molecular structures in their cellular context. (a) slice of a tomogram of a cryo-FIB milled U2OS cell; (b) segmentation of the tomogram in panel a; (c) mammalian nuclear pore complex structure obtained from a single FIB-CET lamellae reproduced from [3]; (d) zoomed in region of (a), red arrows indicate individual membrane proteins (e) CET slice of the nuclear periphery of a mouse- fibroblast. Labels: nuclear pore complex (magenta), ribosomes (cyan), lamina (yellow), chromatin (area inside pink lines; individual nucleosomes can be identified). Scale bars in a,e: $200 \mathrm{~nm}$; c,d: $50 \mathrm{~nm}$. 